\title{
Walking Security Alarm System for Mobile Phone Addicts
}

\author{
Gu Yu ${ }^{1, a}$, Chen Lei ${ }^{1, b}$ and Ji Xiaoyong ${ }^{1, c}$ * \\ ${ }^{1}$ Institute of Electronic Science and Engineering, Nanjing University, Nanjing, 210023, China \\ a15996298013@163.com, bclnjuee2011@163.com, cjxy@nju.edu.cn
}

\begin{abstract}
Keywords: mobile phone addicts; step counting algorithm; fall-down detection algorithm; graded alarm technology

Abstract. To handle walking safety problems for mobile phone addicts, a walking security alarm system based on android mobile platform is designed. According to movement features when someone is walking and when he falls down, this paper proposes a step counting algorithm and a fall-down detection algorithm based on SVM judging respectively. In addition, a graded alarm technology is designed, so that a complete security alarm and calling-for-help system is implemented. Test results indicate that the accuracy of core algorithms have reached over $96 \%$ in average, and functions of each module have reached the design objects.
\end{abstract}

\section{Introduction}

Along with the rapid development of smart phones and mobile technology, mobile phones have become people' $\mathrm{s}$ indispensable portable devices in daily life. In recent years, using mobile phones when bowing down, therefore paying little attention to road traffic conditions, have produced lot of cases causing danger of life. Taking this into account, an APP that can run on mobile phones and issue warnings against users' walking security seems quite urgent. This paper aims at designing a kind of walking security alarm system based on android mobile platform, protecting the security of mobile phone addicts.

\section{Analysis of current technology}

The flaws and blank of current technology are as follows: lack of a real-time step counting algorithm which is easy to calculate and has both high precision and high accuracy; no way to judge whether users are using mobile phones only according to the increasing of step counts, which means we need more factors; lack of a graded alarm mechanism of walking security, and the security protection of mobile phone addicts is in a blank state; lack of an effective fall-down detection algorithm and calling-for-help mechanism when mobile phone users fall down accidentally.

In terms of step counting algorithms, essay[1,2] put forward a kind of step counting algorithm based on the plus and deduction window filter, reducing influences of multi-peak values, and the weak point is that accuracy reduces obviously when walking at variable speed. Essay[3,4,5] put forward a kind of step counting algorithm based on autocorrelation analysis, reducing influences of positions of phones and movement postures. The weak point is that the algorithm is relatively complex and sampling frequency is too low. Essay[6,7,8,9] put forward a kind of step counting algorithm based on calibration factors and delayed feedback, reducing influences of multi-peak values, and its robustness is relatively ideal.The weak point is that accuracy reduces when walking slowly. Essay[10] puts forward a kind of step counting algorithm based on amplitudes of SVM, achieving low power consumption and high accuracy.The weak point is that we need to adjust amplitude threshold due to users' practical movement characteristics, therefore inconvenient in actual use.

In terms of fall-down detection algorithms, essay[11] puts forward a kind of fall-down detection algorithm based on judging SVM and attitude feature thresholds, achieving relatively low missing rate and false rate. The weak point is that threshold parameters are fixed in certain age groups.Essay[12] puts forward a kind of fall-down detection algorithm based on Kalman filtering on body attitude angels, also achieving relatively low missing rate and false rate. The weak point is that the filter algorithm is 
relatively complex. Essay[13] puts forward a kind of fall-down detection algorithm based on Hidden Markov Model training SVM and body attitude, achieving relatively low missing rate and false rate.The weak point is that the training process will take extra time. Essay[14] puts forward a kind of fall-down detection algorithm based on SVM and extracting SMA peaks. The data are easy to collect, but missing rate and false rate are not low enough. All of the above four algorithms use external accelerometer to collect data, and the missing rate and false rate will increase in actual detection, which is harmful to the detection in this part,considering the restriction of built-in accelerometer.

Against deficiencies of current technology and flaws of algorithms, this paper proposes a step counting algorithm and a fall-down detection algorithm based on SVM judging.Both algorithms are simple and efficient, achieving relatively high precision and high accuracy. In combination with the algorithms, we use built-in face detection technology of android mobile phones, implementing real-time and accurate judgment of mobile phone addicts, locking targets efficiently. Also, a graded alarm technology is designed creatively, reflecting humanize designing concept.

\section{System module division}

Module 1: step counting module, responsible for judging whether the user is walking.

Module 2: Face detection module, responsible for judging whether the user is using the mobile phone, therefore judging whether the user is a mobile phone addict right now.

Module 3: Graded alarm module, responsible for issuing warnings to users according to their personal information and current statement.

Module 4: fall-down detection module, responsible for monitoring if the user happens to fall down, calling for help timely when fall-down occurs.

\section{Core algorithm and technology Step counting algorithm}

According to the movement characteristics of human walking, we choose SVM(Signal Vector Magnitude, $\mathrm{SVM}=\sqrt{A_{x}^{2}+A_{y}^{2}+A_{z}^{2}}$ ) as evidence of judging whether steps increase or not, so that the interference from phone movement angle can be excluded.

Acquire acceleration data of $X / Y / Z$ axis, named $A_{x} / A_{y} / A_{z}$, calculate $S V M$, mean filter with the window length of 3 , and draw the waveform.

According to periodic characteristics of the waveform, we try to find out the repeated parts of every period, namely the part where SVM decrease from $A_{1}$ to $A_{2}$, judge whether the user actually takes a step according to $T_{1}$, which refers to the time length this part occupies. With this method we can avoid interference from sensor data jitter or other external factors. As normal walking speed of human stays within a certain range, we should calculate the time interval between two adjacent steps, namely $T_{2}$. After repeated test and revise, we decide the value of $A_{1}$ as $10.5 \mathrm{~m} / \mathrm{s}^{2}, \mathrm{~A}_{2}$ as $9.1 \mathrm{~m} / \mathrm{s}^{2}, \mathrm{~T}_{1}$ as $0.03 \mathrm{~s}, \mathrm{~T}_{2}$ as $0.40 \mathrm{~s}$. Each time when SVM meets the conditions above, the total steps increase 1 . When the total steps increase from 0 to 5 , we judge that the user begins to walk. When the total steps no longer increase over 3 seconds, we judge that the user stops walking, and the total steps will be set zero.

\section{Face detection}

Step1 When the user is detected walking by using the step counting algorithm above, we initialize the front camera, and take a picture every second.

Step2 Store the data obtained from the picture into a temporary bitmap, rotate 90 degree counterclockwise and zoom the bitmap with the proportion of 1:0.3.

Step3 Transfer the bitmap to the format of RGB_565, use the default FaceDetector class to detect the bitmap. When there's complete outline of human face, and the angle between the face and the mobile phone stays within the range of $\pm 45^{\circ}$ on the $\mathrm{X}$ axis, $\pm 30^{\circ}$ on the $\mathrm{Y}$ axis, $\pm 30^{\circ}$ on the XOY plane, then the face will be detected, and the user is judged using the mobile phone. 


\section{Graded alarm}

Step1 Turn on the timer to record the duration. When the user use the mobile phone while walking for more than 15 seconds, start alarm of level 1, pop up a line of warning word with a warning sign: Use the mobile phone while walking for too long may cause danger, please be careful.

Step2 When the user use the mobile phone while walking for more than 30 seconds, start alarm of level 2, pop up a line of warning word with a warning sign: You are walking in the state which may cause danger, please be careful. Then start the vibration function, broadcast the warning voice circularly.

Step3 When the user use the mobile phone while walking for more than 2 minutes, start alarm of level 3, pop up a line of warning word with a warning sign: You've been use the mobile phone while walking for over 2 minutes, the screen will be turned off for your safety in 10 seconds. Then pop up a countdown dialog box, start the vibration function, and broadcast the warning voice circularly.

Step4 When the user stop using the mobile phone while walking for over 5 seconds, then stop alarming, record the level and duration of this alarm, store the data into the database in the mobile phone. When level 3 alarm is triggered three times within 30 minutes, then start 10 seconds countdown, turn off the screen in 10 seconds by controlling the phone power management.

Note: For women users, users aged under 18 or over 60 , and users with movement disorder, level 1 alarm trigger time will be 10 seconds, level 2 alarm trigger time will be 20 seconds, level 3 alarm trigger time will be 60 seconds.

\section{Fall-down detection}

Choose SVM as evidence to judge the mutation of movement state, so that the interference from phone movement angle can be excluded.

Acquire acceleration data of $X / Y / Z$ axis, named $A_{x} / A_{y} / A_{z}$, calculate $S V M$, mean filter with the window length of 3 , and draw the waveform.

According to the waveform characteristics of sudden fall-down in walking process, there will be a short peak in SVM waveform, and the mobile phone will stay still in the next several seconds after the fall-down.

Firstly, according to the feature of SVM be greater than the threshold $\mathrm{A}_{1}$ within a continuous interval $\mathrm{T}_{1}$, mark out the time point where a fall-down is suspected to occur. Then check whether SVM stays within the range between $A_{2}$ and $A_{3}$ in the time interval between $T_{2}$ and $T_{3}$, judge if the mobile phone stays still during the interval, further judge if a fall-down occurs. After repeated test and revise, we decide the value of $A_{1}$ as $15.6 \mathrm{~m} / \mathrm{s}^{2}, \mathrm{~A}_{2}$ as $8.8 \mathrm{~m} / \mathrm{s}^{2}, \mathrm{~A}_{3}$ as $10.8 \mathrm{~m} / \mathrm{s}^{2}, \mathrm{~T}_{1}$ as $0.20 \mathrm{~s}, \mathrm{~T}_{2}$ as $3 \mathrm{~s}, \mathrm{~T}_{3}$ as $6 \mathrm{~s}$. Each time when SVM meets the conditions above, then the user is confirmed to have fallen down unexpectedly. A dialog of inquiry for asking for help will be popped up, and a 5-second countdown will be started at the same time. If the user hasn't cancel the inquiry manually, then begin to alarm by playing the default tone of the mobile phone, and start to vibrate with a short interval. Afterwards, send a message to the emergency number with user's current location information, and call the emergency number or 120 for help.

\section{Experimental evaluation}

Mobile phone used for testing: Huawei 5A

\section{Testing of step counting algorithm}

Horizontal test: Multiple users, hand hold, walking at stable speed(about 95 steps/min)

Longitudinal test: Fixed one user, different posture to hold the phone 
Horizontal Test

\begin{tabular}{|c|c|c|c|c|c|}
\hline Tester & $\begin{array}{c}\text { Steps } \\
\text { measured }\end{array}$ & $\begin{array}{c}\text { Steps in } \\
\text { fact }\end{array}$ & $\begin{array}{c}\text { Steps } \\
\text { measured }\end{array}$ & $\begin{array}{c}\text { Steps in } \\
\text { fact }\end{array}$ & $\begin{array}{c}\text { Average } \\
\text { accuracy }\end{array}$ \\
\hline Student 1 & 52 & 51 & 46 & 50 & $95 \%$ \\
\hline Student 2 & 50 & 50 & 51 & 50 & $99 \%$ \\
\hline Student 3 & 50 & 50 & 51 & 50 & $99 \%$ \\
\hline Student 4 & 50 & 50 & 50 & 50 & $100 \%$ \\
\hline Student 5 & 50 & 50 & 50 & 50 & $100 \%$ \\
\hline Student 6 & 50 & 50 & 50 & 50 & $100 \%$ \\
\hline Student 7 & 51 & 50 & 50 & 50 & $100 \%$ \\
\hline Student 8 & 50 & 50 & 50 & 50 & $100 \%$ \\
\hline
\end{tabular}

Table 1

Longitudinal Test

\begin{tabular}{|c|c|c|c|c|c|}
\hline Walking posture & $\begin{array}{c}\text { Steps } \\
\text { measured }\end{array}$ & $\begin{array}{c}\text { Steps in } \\
\text { fact }\end{array}$ & $\begin{array}{c}\text { Steps } \\
\text { measured }\end{array}$ & $\begin{array}{c}\text { Steps in } \\
\text { fact }\end{array}$ & $\begin{array}{c}\text { Average } \\
\text { accuracy }\end{array}$ \\
\hline Upstairs & 48 & 50 & 50 & 50 & $98 \%$ \\
\hline Downstairs & 48 & 50 & 49 & 50 & $97 \%$ \\
\hline 55 steps/min & 50 & 50 & 50 & 50 & $100 \%$ \\
\hline Variable speed & 49 & 50 & 48 & 50 & $97 \%$ \\
\hline 135 steps/min & 49 & 50 & 47 & 50 & $96 \%$ \\
\hline In coat pocket & 52 & 50 & 52 & 50 & $96 \%$ \\
\hline In pants pocket & 51 & 50 & 50 & 50 & $99 \%$ \\
\hline
\end{tabular}

Table 2

Analysis: Average accuracy of all users when walking at 95 steps/min is $99.1 \%$, while overall accuracy of different posture is $97.6 \%$.

\section{Face detection}

Margin test: The user's face can be detected only when the whole face contour is located in the picture.

Max deflection on $\mathrm{X}$ axis is $\pm 45^{\circ}$ ( with no rotation in XOY plane, no deflection on $\mathrm{Y}$ axis )

Max deflection on $\mathrm{Y}$ axis is $\pm 30^{\circ}$ ( with no rotation in XOY plane, no deflection on $\mathrm{X}$ axis )

Max rotation in $\mathrm{XOY}$ plane is $\pm 30^{\circ}$ ( with no deflection on $\mathrm{X}$ axis, no deflection on $\mathrm{Y}$ axis )

\section{Fall-down detection}

Horizontal test: Multiple users, hand hold, fall down forward when walking at stable speed Longitudinal test: Fixed one user, different posture to fall down 
Horizontal Test

\begin{tabular}{|c|c|c|c|}
\hline Tester & $\begin{array}{c}\text { Fall-down times } \\
\text { tested }\end{array}$ & $\begin{array}{c}\text { Fall-down times in } \\
\text { fact }\end{array}$ & Average accuracy \\
\hline Student 1 & 19 & 20 & $95 \%$ \\
\hline Student 2 & 20 & 20 & $100 \%$ \\
\hline Student 3 & 20 & 20 & $100 \%$ \\
\hline Student 4 & 20 & 20 & $100 \%$ \\
\hline Student 5 & 19 & 20 & $95 \%$ \\
\hline Student 6 & 19 & 20 & $95 \%$ \\
\hline Student 7 & 20 & 20 & $100 \%$ \\
\hline Student 8 & 20 & 20 & $100 \%$ \\
\hline
\end{tabular}

Table 3

Longitudinal Test

\begin{tabular}{|c|c|c|c|c|}
\hline Behavior & $\begin{array}{c}\text { Fall-down or } \\
\text { not }\end{array}$ & $\begin{array}{c}\text { Correct } \\
\text { times }\end{array}$ & Total times & $\begin{array}{c}\text { Average } \\
\text { accuracy }\end{array}$ \\
\hline Lying down still & No & 20 & 20 & $100 \%$ \\
\hline Sitting still & No & 20 & 20 & $100 \%$ \\
\hline Standing still & No & 20 & 20 & $100 \%$ \\
\hline Uniform walking & No & 20 & 20 & $100 \%$ \\
\hline Sit down quickly & No & 20 & 20 & $100 \%$ \\
\hline Fall forward & Yes & 20 & 20 & $100 \%$ \\
\hline Fall Laterally & Yes & 19 & 20 & $95 \%$ \\
\hline Fall backward & Yes & 19 & 20 & $95 \%$ \\
\hline Stand up quickly & Yes & 19 & 20 & $95 \%$ \\
\hline
\end{tabular}

Table 4

Analysis: Average accuracy of all users falling down forward when walking at stable speed is $98.1 \%$, while overall missing/false rate of different posture is $0 / 3.75 \%$.

\section{Conclusions}

This paper elaborates a kind of design of walking security alarm system for mobile phone addicts in detail. In the part of step counting algorithm, the quantity of data collection is reduced, while high precision and high accuracy is maintained. In the part of face detection, we choose default face detecting technology from Google, providing real-time and precise judgement of whether the user is using the mobile phone while walking, so that the target can be locked effectively. According to how long the user has been using the mobile phone when walking, a graded alarm technology is creatively designed, meanwhile special care is given to women, elders, juveniles and users with movement disorder, reflecting humane design concepts while protecting users' walking safety. In the part of fall-down detection, the quantity of data collection is reduced, while high precision and high accuracy is maintained. The whole system has a clear structure and clear division of labor, while every module can play their role according to the designing demand, so that the walking safety of mobile phone addicted users can be fully guaranteed.

The system designed in this paper is part of walking security protecting plan, meanwhile the base of the whole project. We are planning to store real time motion data and location information of every 
user in the cloud, so that we can monitor the user's walking status more effectively, and provide more safeguard. At the same time, we'll do real time image processing for the front road in the user's direction, develop the road image feature database by machine learning, and realize intelligent analysis for the front road condition in the user's direction, so that every user's walking security can be further protected.

\section{References}

[1] Wang W J, LI J. Algorithm design of counting steps based on mobile acceleration sensor [J]. Industrial Control Computer, 2016, 29(1): 75 - 79.

[2] Wu C, Yang Z, Liu Y, et al. WILL: Wireless indoor localization without site survey [J]. Parallel and Distributed Systems, IEEE Transactions on, 2013,24(4): 839 - 848.

[3] Chen G L, Zhang Y Z, Yang Z. Realization of pedometer with auto-correlation analysis based on mobile phone sensor [J]. Journal of Chinese Inertial Technology, 2014, 22(6): 794 - 798 .

[4] Ficco M, Palmieri F, Castiglione A. Hybrid indoor and outdoor location services for new generation mobile terminals [J]. Personal And Ubiquitous Computing, 2014, 18(2): 271-285.

[5] Lan K C, Shih W Y. Using smart-phones and floor plans for indoor location tracking [J]. IEEE Transactions on Human-Machine Systems, 2014, 44(2): 211-221.

[6] Liang J Z, Zhu X J, Chen J. A high-accuracy and low-sampling-rate step counting algorithm design based on smartphone's accelerometer [J]. Journal of Northwest University(Natural Science Edition), 2015, 45(5): 738-744.

[7] Brajdic A, Harler. Walk detection and step counting on unconstrained smartphones [C]. The International Joint Conference on Pervasive and ubiquitous computing. New York: ACM, 2013: 225-234.

[8] Oshin T O, Poslad S. ERSP: An energy-efficient real-time smartphone pedometer [C]. IEEE International Conference on Systems, Man, and Cybernetics(SMC), Manchester: IEEE, 2013: 2067-2072.

[9] Jayalath S, Abhayasinghe N. A gyroscopic data based pedometer algorithm [C]. International Conference on Computer Science \& Education, Colombo: IEEE, 2013:551-555 .

[10] Song H, Zhang R, Wang Z M. Low power consumption pedometer algorithm for mobile phones [J]. Journal of Xi'An University of Posts and Telecommunications, 2016, 21(1): 106-109.

[11] Zhuo C B, Yang L P, Zhou L, Luo D. Design of Fall Detection System Based on MPU6050 Acceleration Transducer [J]. Chinese Journal of Electron Devices, 2015, 38(4): 821-825.

[12] Wang R, Zhang Y, Chen J X. Design and implementation of fall detection system using tri-axis accelerometer [J]. Journal of Computer Applications, 2012, 32(5): 1450-1452 .

[13] Lu X L, Wang H B, Wang Y Y, Xu B G. Human falling detection based on accelerometer [J]. Application Research of Computers, 2013, 30(4): 1109-1111.

[14] Zhang J J, Zhao J, An B J, et al. Triaxial Accelerometer-Based Fall Detection Research [J]. Progress in Modern Medicine, 2014, 14(18): 3585-3588. 